\title{
The development and validation of an ontology of intelligent buildings
}

\author{
T. Gadakari ${ }^{1}$ \& S. Mushatat ${ }^{2}$ \\ ${ }^{1}$ School of Art and Design, Faculty of Arts and Humanities, Coventry \\ University, UK \\ ${ }^{2}$ School of Architecture and Built Environment, University of \\ Wolverhampton, UK
}

\begin{abstract}
An intelligent building can be defined as a dynamic and responsive infrastructure that integrates disparate building systems to effectively manage resources, provide benefits and optimise processes. The need for better knowledge management and development of research requires that intelligent buildings and their systems be classified. This paper develops an ontology of intelligent buildings based on a hierarchical framework, a formal definition of building intelligence and highlights their Key Performance Factors (KPF). A systematic qualitative review approach was used to investigate the current state of knowledge in the field of evolution of intelligent buildings, types of intelligent building technologies (IBT), methods to develop an ontology and the components of an ontology. Then the ontology was created by segregating the various elements of an intelligent building as "objects" and "classes", along with their defining attributes and the relations between them. Some significant examples of coordination among the intelligent building systems were reported. It was deduced that every current building can be considered intelligent, though the degree of intelligence would vary according to the systems used and their attributes. KPFs were identified, which impart intelligent properties to building technologies and thus help decide the level of building intelligence. Subsequently this research was validated by an on-site study of the BRE Innovation Park, UK. Observing seven state-of-art demo buildings equipped with the latest innovations in intelligent building technologies helped assess, if all the necessary features of intelligent buildings were part of the ontology and whether the established hierarchy was consistent with operations observed on-site.

Keywords: BRE Innovation Park, intelligent buildings, ontology, framework.
\end{abstract}




\section{Introduction}

A systematic chronological review of the various definitions [1-9] of intelligent buildings dating from as early as the 1980s to the latest ones revealed that an intelligent building can best be described as a dynamic and responsive infrastructure that integrates disparate building systems such as lighting, HVAC, security, facilities management, etc., to effectively manage resources, provide high performance benefits and optimise processes, comfort, energy costs and environmental benefits. Although there is no standard recognised definition available it was observed that the definition of intelligent buildings had evolved from an early techno-centric one to a more all-encompassing one that focuses on issues such as environmental monitoring, security, communication and people's comfort. Furthermore, speculation of advances in the fields of artificial intelligence (AI), robotics, automation, and architecture, confirm that by 2020, most of the buildings around the world will be increasingly intelligent [4, 10-12]. Hence, it is key that an on-going dialogue for research development in this area is pursued fervently so as to ensure that we gather and classify knowledge about the continuously evolving intelligent buildings so that they are mainstream, practical, and viable.

Scientific progress has always been based on classification. Classification helps bring clarity to the subject as well as structures the study area. The need for better knowledge management and development of research requires that intelligent buildings and their systems be classified. This research aims to contribute to the knowledge of intelligent buildings through the development of its ontology. By developing an ontology this research contributes to: a shared common understanding of the structure of information; enables reuse of domain knowledge; makes domain assumptions explicit; and analyses domain knowledge (Noy and McGuinness [13]).

A systematic qualitative review approach was used to investigate the current state of knowledge in the field of intelligent buildings, especially the various types of intelligent building technologies (IBT), what they constitute of, and how they interact with each other. The methods to develop an ontology and the components of an ontology were studied. Then the ontology was created by segregating the various elements of an intelligent building such as its various systems (lighting, HVAC, security, integrated building automation, etc.), their types, properties and interrelationships. Subsequently the ontology was validated by an on-site study of the BRE Innovation Park, at Watford, UK, which is a state-of-art demonstration site that is constantly being updated according to the latest innovations in intelligent and sustainable building technologies. The purpose of the study was to observe the various types of IBTs used in the seven demonstration buildings and assess if all the necessary components of intelligent buildings were a part of the ontology and whether the established hierarchy was consistent with operations observed on-site. 


\section{Ontology of intelligent buildings}

Gruber [14] reports, an ontology formally represents knowledge as a hierarchy of concepts within a domain, using a shared vocabulary to denote the types, properties and interrelationships of those concepts. Smith [15] describes ontology as the first approximation in scientific classification, a table of categories. Ontologies are the structural frameworks for organising information and are widely used as a form of knowledge representation in artificial intelligence, Semantic Web, systems and software engineering, biomedical informatics, library science, enterprise bookmarking, and information architecture [14, 16]. In practical terms, developing an ontology includes: defining classes in the ontology; arranging the classes in a taxonomic (subclass-superclass) hierarchy; and describing the relations between the classes. Components of an ontology include: Objects (individuals or instances); Classes (sets, collections, concepts, types of objects, or kinds of things); Attributes (aspects, properties, features, characteristics, or parameters that objects and classes have); and Relations (ways in which classes and individuals are related to one another) [13, 15].

The current state of knowledge in the areas of evolution of intelligent buildings; types of IBTs; and the anatomy of intelligent buildings was reviewed (journal articles; books; online and magazine articles; government and private organisation reports). The observations from the literature review suggested, that intelligent buildings can be categorised as: the various building control systems that can act autonomously such as lighting, HVAC and security; the building blocks of these systems such as sensors and controllers; and other advanced systems that can integrate and manage.

\subsection{Building control systems}

The literature review highlighted that intelligent buildings comprise of autonomous subsystems which can be programmed to collaborate with the aid of advanced systems (Section 2.2). Some of these systems are described below.

\subsubsection{Lighting systems}

Intelligent lighting systems can complete an array of functions ranging from turning a light on/off to adjusting light and shade levels to completely managing the lighting through an IT backbone [17]. Some examples include: lighting with occupancy sensors for presence detection; lighting with daylight sensing photo sensors to adjust indoor lighting based on natural light conditions; automated shading system that adjusts the angle of blinds based on external parameters such as temperature and sunlight.

\subsubsection{HVAC control systems}

Intelligent HVAC systems can improve the indoor air quality by monitoring the temperature, humidity or by adjusting the number of air changes according to the number of occupants by using temperature, moisture and $\mathrm{CO}_{2}$ sensors respectively [18]. It can also save energy by using programmable zones that manage HVAC only in occupied areas, or automatically shut off when natural 
ventilation is introduced. Louvres of windows/doors can be controlled by temperature and $\mathrm{CO}_{2}$ sensors, and carbon monoxide sensors linked to pulse fans in car parks provide fresh air and get rid of undetectable poisonous gases.

\subsubsection{Security systems}

Intelligent security systems carry out a range of activities and can be classified as access control systems (using smart card, proximity cards or biometric details), surveillance systems (e.g. cameras with motion and facial detection), tracking systems (e.g. smart carpeting that tracks individuals and activity), digital data security systems. Some other interesting examples include a Visitor Management System and a Guard Tour System that tracks visitors and manual security inspections at frequent intervals respectively [4, 18-21].

\subsubsection{Fire and life safety systems}

According to Harrison et al. [4] a major trend in intelligent fire systems is based on a greater reliability in fire detection and protection. Integrated Fire Alarm systems have connections between fire alarm sensors and the fire alarm panel to accurately locate the fire; activate shutdowns, evacuation systems and addressable sprinkler systems that only target the affected area; and coordinate other building systems for safety [10]. There are other life safety systems such as gas leak detection sensors, earthquake sensors and flood detection sensors.

\subsubsection{Feedback and display systems}

Wacks [6] describes feedback and display systems as being used to inform and educate building occupants about relevant data associated with the building such as environmental and operational data, signages and interfaces. The digital signage content predominantly displays information relevant to the live operational metrics gathered during operations, room allocations, control options and other data.

\subsection{Integrative building systems}

A key characteristic of intelligent buildings is subsystem synergies. In order to make a building truly responsive and intelligent, the infrastructure services of each subsystem need to be monitored and controlled through a common network that forms a homogeneous and interoperable system. Aristotle said "the whole is greater than the sum of its parts" which holds true in the context of intelligent buildings. The literature has suggested that the independent subsystems (Section 2.1) with the aid of integrative building systems should be interconnected.

\subsubsection{Building Automation System (BAS)}

The BAS comprises of the Building Management System (BMS), Energy Management System (EMS) and Facilities Management System (FMS).

The BMS deals with centrally controlling and monitoring the working of all the building systems in tandem to promote efficiency. A competitive BMS should be: an advanced open system, respecting common standards and various manufacturers; easy to install in existing buildings without a major retrofitting 
effort; and designed in such a way that essential services continue to be delivered in the default stand-alone mode in case of emergency situations [5, 6].

The EMS coordinates all the energy-consuming autonomous building systems to operate in harmony. The EMS should be as dynamic as possible in order to respond to changing power, cooling and heat loads. EMS should intelligently manage the various power states necessary to optimise power consumption i.e. only the components necessary to perform a function at any given time are running at full power, while other components are on standby or in a disconnected state [3, 22].

An FMS unifies autonomous building systems to work together in an automated manner that dynamically responds to scheduled events and occupancy, in order to achieve the highest level of energy efficiency and user experience. A FMS allows easy access to building systems, remote monitoring of the facility and provides automated work orders for property managers. It also empowers the operations team with the tools to run the building in the most practical manner $[4,22]$.

\subsubsection{Integrated Communication Systems (ICS)}

An ICS enables building systems to share data among themselves and integrates them using web-enabled integration platforms, IP-enabled devices, communication protocols, local area networks, shared networks, telecommunications and a flexible IT backbone. An efficient ICS should process data and information on a robust data network and be responsive to change.

\subsection{Building blocks of intelligent buildings}

\subsubsection{Sensors}

Wilson [23] regards sensors as the interface between the physical world and the world of electrical devices. They measure multiple physical properties and provide input data to the controllers. They include movement or occupancy sensors, temperature and humidity sensors, light-level sensors, electronic sensors, chemical and biosensors, status sensors and tagging systems [22, 24].

\subsubsection{Controllers}

Controllers are purpose-built computers with input and output capabilities which control devices found in building systems. In case of an autonomous subsystem, the data gathered by the different sensors is used locally through actuators or in case of connection to a BAS it is coordinated with other subsystems through a gateway [25]. Inputs allow a controller to read temperatures, humidity, etc. and send control signals to devices, and other parts of the system.

\subsubsection{Actuators}

An actuator is the mechanism by which a control system acts upon an environment. Their task is to convert the electric signal received into a physical phenomenon. It is a type of motor operated by a source of energy that is responsible for moving or controlling a system. 


\subsubsection{User interface (UI)}

The user interface helps the users control the various building subsystems. When a subsystem is autonomous it usually has an independent UI but when it is connected to the BAS the UI is centrally managed through the network. Though in the latter case provisions can be made for the UI to be made available for personalised control of the space so that users feel in control and at ease [26, 27].

\subsection{Ontology development}

The ontology of intelligent buildings was created using its various systems, their types, properties and interrelationships. It focuses on the relationship between these automated systems, in terms of their hierarchy and their efficient integration and communication with each other. The literature review enabled the classification of an intelligent building as objects and classes in the form of a systematic hierarchical framework (Figure 1).

- Objects: Objects are the ground level parts of an intelligent building and are the building blocks of subsystems. They include sensors, controllers, actuators and user interfaces.

- Class II (Sub-class): Class II systems exist in an intelligent building either autonomously or can have the ability to interact with each other with the aid of a Class I system. They comprise of “objects”.

- Class I (Super-class): The intelligence of system coordination is logically located at a layer above the Class II systems. This intelligent layer of system coordination can be described as the Building Automation System (BAS) and the Integrated Communications System (ICS). The BAS typically comprises of the BMS for central control and monitoring; EMS for controlling and monitoring energy usage; and FMS for efficient day-today working and maintenance. The ICS integrates all or some of the subsystems so that they can be managed by the BAS.

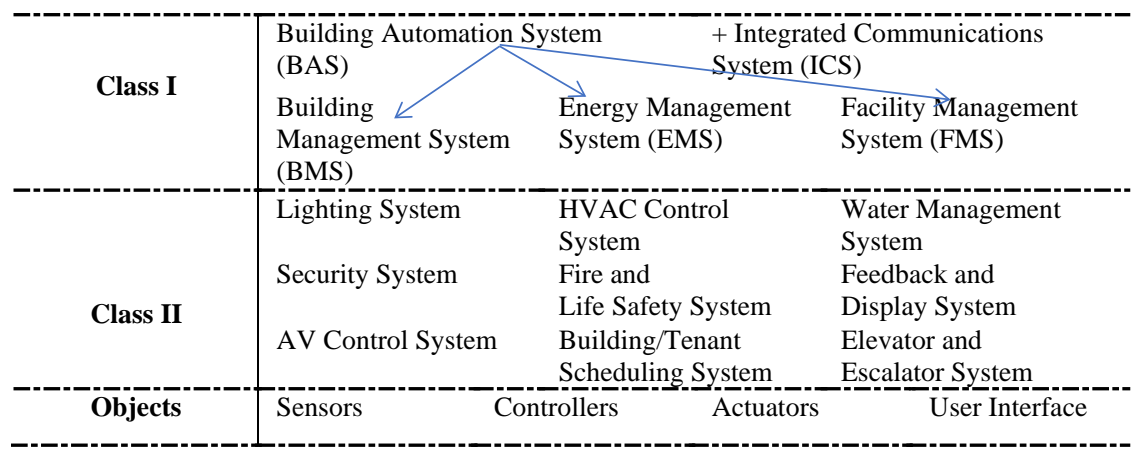

Figure 1: The ontology of intelligent buildings. 


\subsection{Examples of relations between objects and classes}

Section 2.5 highlights some of the important examples of coordination among the various classes and objects that ensure that the intelligent building "knows", “decides" and "responds" dynamically to its environment.

- HVAC control system, security system and lighting system interaction: When an employee comes to work out of hours, a swipe of their key card in the access-control system for entry, triggers the heating and lighting in the office area to power up, such that the space is welcoming on arrival. Also the outdoor airflow to that area can be increased and window shades can be opened. Security cameras can be cued, only the doors to the area where the employee works can be unlocked automatically while other doors remain locked, and elevator security configurations can be changed to allow access to certain floors only, for enhanced security purposes.

- Fire and life safety system's interaction with other systems: During a fire situation, the alarms sound which leads to other systems to react - exhaust dampers open, the lighting system flashes warnings to evacuate, the IP intercom system issues instructions to occupants, the access-control system unlocks doors for evacuation, and CCTV cameras provide emergency responders with a view of the fire.

- HVAC control system and security system interaction: By integrating demand-response ventilation system with video cameras the information can help calculate the building's occupancy, and the ventilation system's VAV terminals can then distribute an appropriate amount of outside air.

- Lighting, HVAC and AV control systems integrated with building/tenant scheduling systems: Systems remain in low-power mode until the scheduling system indicates that the room is in use, which can trigger power-up sequencing. Occupancy sensors can be used to inform the scheduling application if the room is vacant, even though it is scheduled, so the room may be made available again in the scheduling system. Systems can power down and HVAC set-points changed when a room is unscheduled. When a room is in an unscheduled state, the lights and AV system shall be 100 percent off, the HVAC set-point adjusted and shades adjusted to reduce or leverage heat gain as appropriate.

\section{Case study of the BRE Innovation Park}

The BRE Innovation Park in Watford is the Building Research Establishment's (BRE) attempt to showcase some of the world's most sustainable buildings through the use of emerging and innovative technologies, including those, which have not yet reached the marketplace [28]. It was chosen as a case study for validation of the ontology as it showcases the current state-of-art in intelligent building technologies. By observing the demonstration buildings equipped with the latest innovations helped assess, if all the necessary features of intelligent buildings were part of the ontology and whether the established hierarchy was 
consistent with operations observed on-site. The site was congenial and accessible but most importantly it was a huge repository of knowledge.

The case study visit began at the Visitor Centre that provides information on all the buildings and innovative new design products at the park. It also houses the Digital Management Centre which monitors energy use in six homes on campus through the use of smart meters and also integrates their automation. The Smart Home project demonstrates an "intelligent whole house living system" that enables control using occupation sensors for a range of purposes to control and monitor the heating, lighting, ventilation, security, and water and energy use. It can also monitor the movements of the elderly and make sure that they are safe. The Willmott Dixon Community Healthcare Campus showcases the latest innovations in healthcare technology. Some examples include intelligent lighting systems that support the human circadian rhythm, mechanical ventilation system with heat recovery options, natural ventilation e-stack system that is automatically controlled by temperature and $\mathrm{CO}_{2}$ sensors, a digital feedback system, BMS and EMS. The Sigma Home or Rexel Innovation Centre showcases and tests the most innovative technologies and low energy products. It has the most number of technologies on display on campus such as an automated solar stack system that controls temperature in the house as dictated by the climatic conditions outside, a BMS, an EMS with a user awareness system, a FMS, a self-monitoring grey-water recycling system, etc. The BRE Victorian Terrace (built in 1855 and extended in 1878 and 1903) is a "living laboratory" to demonstrate advanced control systems that deal with overheating, ventilation, assisted living, lighting, smart meters, and health and wellbeing, as well as optimise energy use in the house. The Hanson EcoHouse demonstrates how sustainable homes can use traditional materials while using smart technologies such as an OpenHub home management system that controls the HVAC and security systems; an EDF energy management system; and an ICS with a full portfolio of home based data services. The Barratt Green House among a range of innovative technologies boasts of a computerised control system ensuring optimal operation of all building services and provides a central data storage facility [28].

After conducting a detailed study of the BRE Innovation Park the different IBTs observed on-site in the various buildings have been reported in Table 1 . Table 1 confirms the presence of the different types of IBTs that were highlighted in the literature review at the state-of-art demonstration site. A number of Class I and Class II systems as categorised in the ontology were observed on site. The different parameters by which the different systems functioned and interacted, reiterated the hierarchy set out by the ontology in Figure 1. Class I systems such as the BMS, EMS, FMS and ICS were clearly placed a layer above the Class II systems and facilitated their efficient functioning. The Objects defined in the ontology were not physically visible as they were essentially software platformbased except for some sensors and user interfaces. One of the interesting facts highlighted by a member of research staff on site was the presence of "pseudoelectrical switches" in the Hanson EcoHouse. These were introduced as most of the systems in the house are automatically controlled by the advanced BMS and 
the people who occupied the house for a short study period did not feel "in control" in the absence of physical switches.

Table 1: $\quad$ Observations at the BRE Innovation Park.

\begin{tabular}{|c|c|c|c|c|c|c|c|}
\hline $\begin{array}{c}\text { Type of intelligent building } \\
\text { technologies }\end{array}$ & 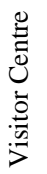 & 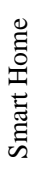 & 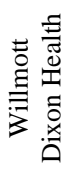 & 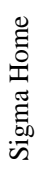 & 呇 & 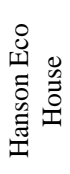 & 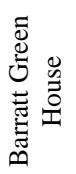 \\
\hline Lighting system & $\sqrt{ }$ & $\sqrt{ }$ & $\sqrt{ }$ & $\sqrt{ }$ & & & $\sqrt{ }$ \\
\hline HVAC control system & & $\sqrt{ }$ & $\sqrt{ }$ & $\sqrt{ }$ & $\sqrt{ }$ & $\sqrt{ }$ & $\sqrt{ }$ \\
\hline Water management system & $\sqrt{ }$ & & & $\sqrt{ }$ & & & \\
\hline Security system & & $\sqrt{ }$ & & $\sqrt{ }$ & $\sqrt{ }$ & $\sqrt{ }$ & \\
\hline Fire and life safety system & & & & $\sqrt{ }$ & & & \\
\hline AV control system & & & & & & & \\
\hline Feedback and display system & $\sqrt{ }$ & $\sqrt{ }$ & $\sqrt{ }$ & & $\sqrt{ }$ & & \\
\hline Tenant scheduling system & & & & & & & \\
\hline Building management system & $\sqrt{ }$ & $\sqrt{ }$ & $\sqrt{ }$ & $\sqrt{ }$ & $\sqrt{ }$ & $\sqrt{ }$ & $\sqrt{ }$ \\
\hline Energy management system & $\sqrt{ }$ & $\sqrt{ }$ & $\sqrt{ }$ & $\sqrt{ }$ & $\sqrt{ }$ & $\sqrt{ }$ & \\
\hline Facilities management system & $\sqrt{ }$ & $\sqrt{ }$ & $\sqrt{ }$ & $\sqrt{ }$ & $\sqrt{5}$ & & $\sqrt{ }$ \\
\hline Integrated communication system & $\sqrt{ }$ & & $\sqrt{ }$ & $\sqrt{ }$ & $\sqrt{ }$ & $\sqrt{ }$ & $\sqrt{ }$ \\
\hline Any other/s & & $\sqrt{ }$ & $\sqrt{ }$ & & $\sqrt{ }$ & & \\
\hline
\end{tabular}

Assisted living systems and telecare systems that monitor and learn the movements of the elderly or disabled to help them in emergencies and daily activities were observed in the BRE Victorian Terrace Assisted Living zone, Willmott Dixon Healthcare campus and at the Smart Home. These were reported in the "Any other/s" category (Table 1), which suggested that they had not been considered while forming the ontology and were not a part of the descriptive framework. Assisted independent living is fast gaining popularity and care needs to be taken to include it to the ontology of intelligent buildings either as an addition to the Fire and Life Safety System category or as a completely new sub-category of its own under Class II. This is an example of future-proofing the ontology, such that new intelligent building technologies of the future could be encompassed within the broad sub-categories or added as a new category, where applicable.

\section{Discussion}

Through the development and validation of the ontology, the key performance factors of building intelligence and a formal definition were identified.

\subsection{Key Performance Factors (KPF) of building intelligence}

It is evident that currently buildings employ a wide range of intelligent building technologies and it can be deduced that every building today can be considered "intelligent", though the degree of intelligence varies according to the systems 
they employ and their attributes. The Key Performance Factors (KPF) can be defined as these qualities, which help decide the level of intelligence of individual objects and classes, as well as their composition as a whole. Keeping this in mind, the level of intelligence of intelligent buildings can be evaluated with regards to its KPFs, namely: Interoperability and Information Exchange (How many systems are connected together and how efficiently do they operate); Connectivity: Level of Integration and Interaction (within systems and with users); Operation Protocol (Manual versus Pre-set Algorithm versus Dynamic Adaptation to operating environment); Self-Awareness (ability to make decisions and be responsive); SelfMonitoring capabilities; Resource Efficiency (in terms of energy, water, $\mathrm{CO}_{2}$, etc.); Convenience and Comfort; and Safety and Security.

\subsection{A formal definition of intelligent buildings}

This definition of intelligent buildings acts as a formal classifier of knowledge for future research so that intelligent buildings can be defined based on their multiple components and their relationships instead of vague abstractions. This definition aims to help future researchers identify what is intelligent and what is not when studying and comparing buildings, much like a check list.

Intelligent buildings (i) are defined as

$i$ = Building Management System + Energy Management System + Facility Management System + Integrated Communication System

Controlling * Integrating * Monitoring

Lighting System + HVAC Control System + Water Management System + Security System + Fire \& Life Safety System + Feedback \& Display System + AV Control System + Building/Tenant Scheduling System + Elevator and Escalator System

Figure 2: A formal definition of intelligent buildings.

\section{Conclusions}

To conclude, as intelligent building technologies evolve and develop, the analysis of intelligent building systems and measurement of their intelligence will continue to be viewed as a vast area of interest. This research is an important stepping-stone towards extending and elaborating the knowledge of intelligence in buildings. This paper has reviewed key concepts pertaining to intelligent buildings and their components to develop an ontology that helps manage and classify knowledge as well as formally defines intelligent buildings so as to lay the ground rules for subsequent research in this area. It will also demarcate what comprises an intelligent building as opposed to what does not, and thus help future research strategy. The hierarchical classification of intelligent buildings identified key performance factors, which attribute intelligent properties to building technologies, thus setting the foundation for a meticulous examination of intelligent buildings from the perspective of what makes them intelligent. From a 
practical perspective, it provides a way for design teams to value the level of intelligence of building systems before strategically choosing the ones that make up an efficient building. It can help set up industry standards in the future, which clients can refer to and decide the best suited intelligent building systems for their design and organisational needs.

This ontology has been developed based on the comprehensive knowledge we currently have of intelligent buildings. Implications from the study of intelligent buildings highlight their ever-evolving nature which warrants continuous further exploration and upgradation of the ontology to accommodate future technologies.

\section{References}

[1] Caffrey, R., The intelligent building, an ASHRAE opportunity. ASHRAE Technical Data Bulletin, 4(1), 1985.

[2] Atkin, B., Progress towards intelligent building. Intelligent BuildingsApplications of IT and Building Automation to High Technology Construction Projects, ed. B. Atkin, Unicom Seminars Ltd: London, 1988.

[3] Clements-Croome, T.D.J., What do we mean by intelligent buildings? Automation in Construction, 6(5-6), pp. 395-400, 1997.

[4] Harrison, A., Loe, E. \& Read, J., Intelligent Buildings in South East Asia, Taylor \& Francis Routledge: London, 1998.

[5] Sharples, S., Callaghan, V. \& Clarke, G., A multi-agent architecture for intelligent building sensing and control. Sensor Review, 19(2), pp. 135-140, 1999.

[6] Wacks, K.P., Best-Practices Guide for Evaluating Intelligent Building Technologies, CABA North America's Home \& Building Automation Association: Ottawa, 2002.

[7] Arup, Arup: Intelligent buildings. www.arup.com/communications/ knowledge/intelligent.htm.

[8] Chen, Z., Facilities intelligence and evaluation: A multi-criteria assessment approach. Energy and Buildings, 42(5), pp. 728-734, 2010.

[9] Peluffo, M., Defining todays intelligent building. www.commscope.com/ Blog/Defining-Todays-Intelligent-Building/.

[10] Nikolaou, T., Kolokotsa, D. \& Stavrakakis, G., Introduction to intelligent buildings. A Handbook for Intelligent Buildings, SMART Accelerate: Athens, 2004.

[11] Kurzweil, R., The Singularity is Near: When Humans Transcend Biology, Penguin Group: New York, 2005.

[12] Mourad, M.M., Ali, A.H.H., Ookawara, S., Abdel-Rahman, A.K. \& Abdelkariem, N.M., An energy-efficient smart home for new cities in Egypt. WIT Transactions on Ecology on the Built Environment: EcoArchitecture V, vol. 142, WIT Press: Southampton and Boston, pp. 115126, 2014.

[13] Noy N.F. \& McGuinness, D.L., Ontology Development 101: A Guide to Creating Your First Ontology, Stanford Knowledge Systems Laboratory 
Technical Report KSL-01-05 and Stanford Medical Informatics Technical Report SMI-2001-0880: Stanford, 2001.

[14] Gruber, T.R., A translation approach to portable ontology specifications. Knowledge Acquisition, 5(2), pp. 199-220, 1993.

[15] Smith, B., Ontology and information systems. The Blackwell Guide to the Philosophy of Computing and Information, ed. L. Floridi, Blackwell Publishing: Oxford, pp. 153-166, 2003.

[16] Jurisica, I., Mylopoulos J. \& Yu, E., Using ontologies for knowledge management: An information systems perspective. Proceedings of Annual Conference of the American Society for Information Science, ASIS: Washington, DC, 1999.

[17] Newsham, G., Mancini, S., Veitch, J., Marchand, R., Lei, W., Charles, K. \& Arsenault, C., Control strategies for lighting and ventilation in offices: Effects on energy and occupants. Intelligent Buildings International, 1(2), pp. 101-121, 2009.

[18] ABB, Smart home and intelligent building control. ABB Group Automation and Power Technologies. www.abb.com/.

[19] Frost \& Sullivan, The Bright Green Buildings - Convergence of Green and Intelligent Buildings, Continental Automated Buildings Association (CABA): Ottawa, 2009.

[20] Luo, R., Lin, T. \& Su, K., Multisensor based security robot system for intelligent building. Robotics and Autonomous Systems, 57(3), pp. 330-338, 2009.

[21] Moran, S. \& Nakata, K., Analysing the factors affecting users in intelligent pervasive spaces. Intelligent Buildings International, 2(1), pp. 57-71, 2010.

[22] Wu, S. \& Noy, P., A conceptual design of a wireless sensor actuator system for optimizing energy and well-being in buildings. Intelligent Buildings International, 2(1), pp. 41-56, 2010.

[23] Wilson, J., Sensor Technology Handbook, Elsevier: Oxford, 2008.

[24] Weber, V., Smart Sensor Networks: Technologies and Applications for Green Growth, Organisation for Economic Co-operation and Development: Denmark, 2009.

[25] Verdone, R., Dardari, D., Mazzini, G. \& Conti, A., Wireless Sensor and Actuator Networks, Academic Press Elsevier: London, 2008.

[26] Baird, G. \& Lechat, S., Users' perceptions of personal control of environmental conditions in sustainable buildings. Architectural Science Review, 52(2), pp. 108-116, 2009.

[27] Chappells, H., Comfort, well-being and the socio-technical dynamics of everyday life. Intelligent Buildings International, 2(4), pp. 286-298, 2010.

[28] BRE, BRE Innovation Park: Part of the BRE Innovation Parks Network. Building Research Establishment Ltd. http://ipark.bre.co.uk/parks/england. 